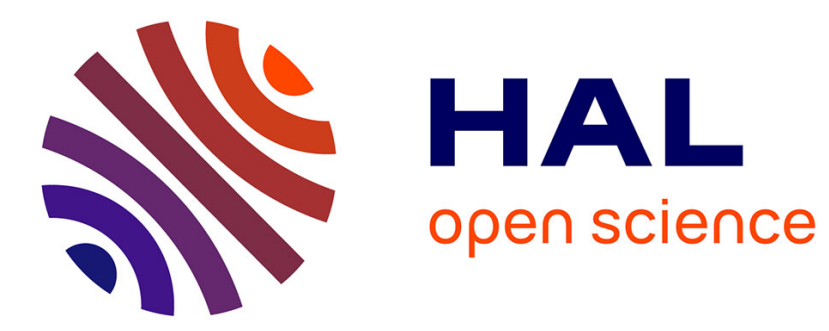

\title{
Hyperbolic K-means for traffic-aware clustering in cloud and virtualized RANs
}

\author{
Hanane Djeddal, Liticia Touzari, Anastasios Giovanidis, Chi-Dung Phung, \\ Stefano Secci
}

\section{- To cite this version:}

Hanane Djeddal, Liticia Touzari, Anastasios Giovanidis, Chi-Dung Phung, Stefano Secci. Hyperbolic K-means for traffic-aware clustering in cloud and virtualized RANs. Computer Communications, 2021, 176, pp.258-271. 10.1016/j.comcom.2021.06.021 . hal-03109662v2

\section{HAL Id: hal-03109662 \\ https://hal.science/hal-03109662v2}

Submitted on 9 Feb 2021 (v2), last revised 13 Jul 2021 (v3)

HAL is a multi-disciplinary open access archive for the deposit and dissemination of scientific research documents, whether they are published or not. The documents may come from teaching and research institutions in France or abroad, or from public or private research centers.
L'archive ouverte pluridisciplinaire HAL, est destinée au dépôt et à la diffusion de documents scientifiques de niveau recherche, publiés ou non, émanant des établissements d'enseignement et de recherche français ou étrangers, des laboratoires publics ou privés.

\section{(ㅇ)(1) 80}

Distributed under a Creative Commons Attribution - NonCommercial - ShareAlikel 4.0 


\title{
Hyperbolic K-means for traffic-aware clustering in cloud and virtualized RANs
}

\author{
Hanane Djeddal ${ }^{\mathrm{a}}$, Liticia Touzari ${ }^{\mathrm{a}}$, Anastasios Giovanidis ${ }^{\mathrm{a}, \mathrm{b}}$, Chi-Dung Phung ${ }^{\mathrm{c}}$, Stefano \\ Secci $^{\mathrm{c}}$ \\ ${ }^{\text {a }}$ Sorbonne University, ${ }^{b}$ CNRS-LIP6, 4 Place Jussieu, Paris CEDEX 05, France, 75252 \\ ${ }^{\mathrm{c}}$ Cnam, Cedric Lab, 292 Rue Saint-Martin, Paris, France, 75003
}

\begin{abstract}
As the internet and connected objects gain more and more in popularity, serving the ever-increasing data traffic becomes a challenge for the mobile operators. The traditional cellular radio access network (RAN), where each base station is co-located with its own processing unit and is responsible for a specific geographic area, has evolved first with the so-called Cloud RAN (C-RAN), and is currently undergoing further architectural evolution under the virtualized RAN (vRAN), Open RAN (O-RAN) and Software-Defined RAN (SD-RAN) architectures. In all these versions, the data processing units can be dynamically centralized into a pool and shared between several base stations, enlarging the geographical view for scheduling and resource allocation algorithms. For instance, resource utilisation is improved by avoiding resource idling during off-peak hours. C-RAN and vRAN gains depend strongly on the clustering scheme of radio units (RRHs and RUs). In this paper, we propose a novel radio clustering algorithm that takes into account both the traffic demand and the position of stations, by using the hyperbolic distance in 3dimensions. We introduce a modified K-means clustering algorithm, called Hyperbolic K-means, and show that this generates geographically compact RU clusters with traffic charge equally shared among them. Application of our algorithm on real-world mobile data traffic, collected from the cities of Nantes and Lille in France, shows an increase in resource utilisation by $25 \%$, and a reduction in deployment cost by $15 \%$, compared to the standard RAN. Furthermore, the performance of our Hyperbolic K-means algorithm is compared extensively against alternative C-RAN clustering proposals from the literature and is shown to outperform them, in resource utilisation as well as in cost reduction.
\end{abstract}

Keywords: C-RAN, vRAN, O-RAN, SD-RAN, K-means, Clustering, Hyperbolic geometry, Poincaré half-plane.

\section{Introduction}

With the rapid growth in consumer electronics, boosted by the Internet-of-Things, more and more wireless products are entering the market adding to the total number of devices accessing mobile networks. According to Cisco 2017, ${ }^{\text {Cisco, } 2017}$ the share of smart devices and connections from the total of connected objects will increase from 53\% in 2017 to $73 \%$ by 2022 . Furthermore, new services emerge related to Internet-of-Vehicles, remote control, and video monitoring, China-Unicom, 2017 which are expected to generate intensive demand for network edge-computing resources. Satisfying such increase in traffic presents a big challenge for mobile operators; a naive installation of more base stations or addition of more data processing units to existing sites, has high deployment and energy expenses, without guaranteed service improvement or revenue increase for the mobile operators.

As an alternative solution for low cost, and low energy-consumption, around 2010 the Cloud Radio Access Network (C-RAN) architecture was conceived by IBM ${ }^{\text {Lin et al., } 2010}$ and developed by the China Mobile Research Institute, ${ }^{\text {China-Mobile, } 2011}$ later adopted also by other operators. This concept is currently enlarged to accommodate various other possible configurations with the socalled virtualized RAN (vRAN), which offers flexibility in functional splitting for 5G architectures, Garcia-Saavedra et al., 2018 , Maeder et al., 2014 da Silva Coelho et al., 2020 
While in the traditional RAN, illustrated in Fig. 1a, each site has its own Remote Radio Head (RRH) and Base-Band Unit (BBU), in the C-RAN architecture base stations (BS) host only RRHs whereas the BBUs are centralized in a pool allowing resources to be dynamically allocated to the RRH cluster, Checko et al., 2015I et al., 2014 see Fig. 1b. By removing BBUs from cell sites, operating expenses as well as energy consumption can be reduced, while providing the same coverage and a better quality of service, Wu et al., 2015 . Boulos et al., 2015

Current vRAN systems are split into three parts:, Wang et al., 2016, Wang et al., 2017Yu et al., 2020 the Radio Units (RU) are geographically scattered to achieve coverage; these are connected to Distributed Units (DU), where some layer-1 and layer-2 functions can be performed; the latter are in turn connected to Centralized Units (CU) where base-band operations can be run, along with other RAN or-

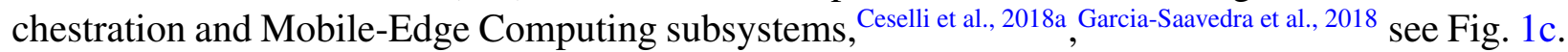
The C-RAN system can be seen as a special case or just an application of a vRAN, where vBBUs are hosted at CUs, and RRH correspond to the RU and DU combined. Generally, RU and DU are expected to be collocated or geographically be very close to each other in practice, and transport links provide connections between the DUs and the $\mathrm{CU}$ hosting the BBU assigned to the RU-DU subsystem. In 5G and beyond 5G systems, it is envisioned that CU, BBU and MEC servers can be co-located at different edge network sites, typically the so-called Central Office (CO), legacy net-

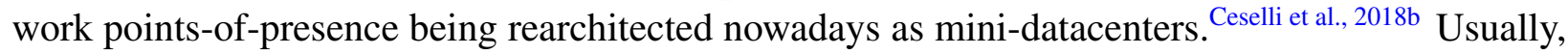
the distance between an RRH and its BBU-pool (resp. DU and CU) can reach several kilometers and fiber lines are used for reliable high bandwidth data transfer.

In the following, we will mostly focus on the C-RAN application but the algorithm we propose can be adapted to vRAN/MEC as well, where base stations are clustered at the CU level. We will use the abbreviation RU to refer to both RRH (for C-RAN) and RUs (for vRAN).

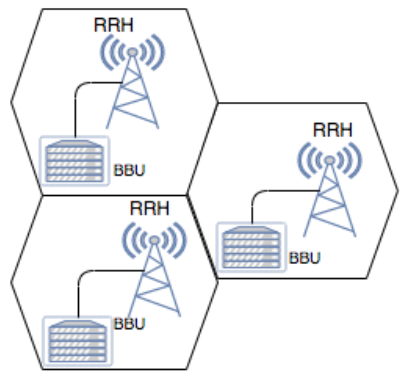

(a) Traditional RAN architecture

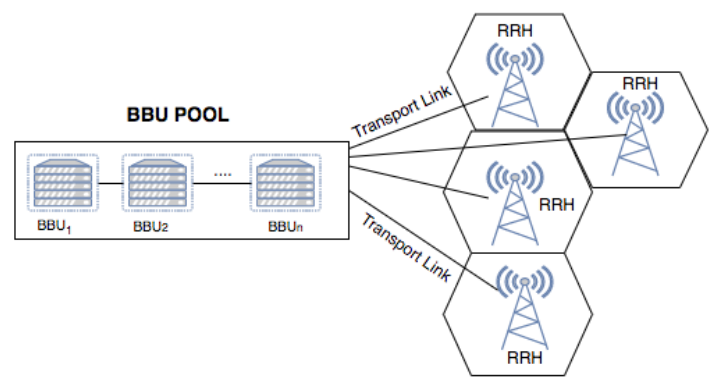

(b) C-RAN architecture

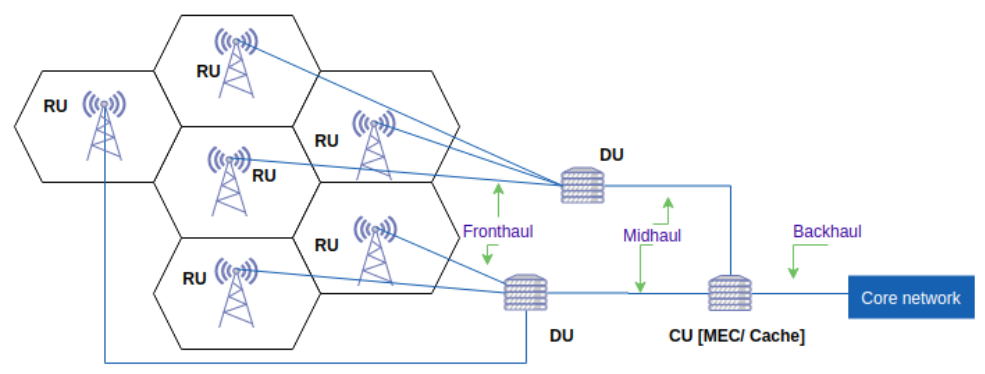

(c) vRAN architecture

Fig 1: Illustration of the traditional RAN architecture and comparison with C-RAN and vRAN. 
Aggregating resources to a pool level allows them to be managed more efficiently and optimize their utilisation. Each BBU pool is in charge of several RUs, hence the installed capacity of the pool should be enough to serve the maximal ensemble traffic generated/received by these RUs, and the total capacity can be further shared among them according to their real-time demand. Performance is optimal when the BBU-pool utilisation is as flat as possible through the day, with a peak-to-average ratio close to one. To achieve this, clustering based on the traffic pattern of the stations (RUs) can be exploited. In fact, grouping RUs with complementary traffic patterns to the same BBU-pool can reduce the total capacity necessary to support the aggregate traffic of the

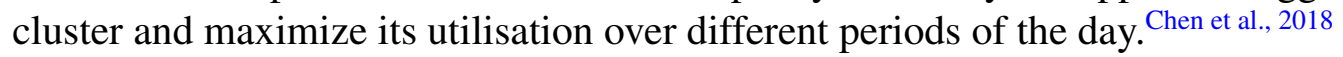

A meaningful clustering scheme should then, (i) reduce the overall processing (BBU) capacity necessary and maximize its utilisation. (ii) The load should be fairly balanced among clusters. The scheme should also (iii) keep a limit on the optical fiber distance between the BBU-pool and its assigned RUs in order to respect delay and propagation constraints. Furthermore, (iv) the clusters should be geographically as compact as possible, with neighbouring RUs assigned to the same cluster, in order to avoid frequent hand-overs between clusters when users are mobile.

In this paper, we propose a BBU-RUs clustering solution that offers improved capacity utilization and reduced deployment cost, while respecting geographic requirements and load fairness among clusters. Our starting point is the standard K-means algorithm, but we introduce a nontrivial modification of the distance metric. The proposed modification involves the distance of the Poincare half-plane model in 3-dimensions ("hyperbolic $\mathbb{H} 3$ "), which allows us to appropriately combine the different natures of traffic and positional features. This approach was motivated by

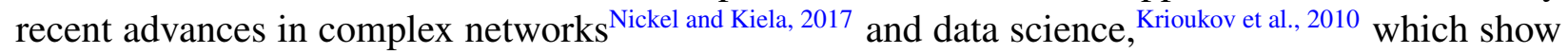
that embedding complex data into hyperbolic spaces is very advantageous for problems of clustering and community detection. Gerald et al., ${ }^{2020}$ Our method, being center-based, groups RUs around a fictional center (so-called "centroid") which determines the average charge of the cluster. The goal is to end up with a fair clustering scheme, which satisfies the above design requirements (i)-(iv). The solution is done per time-slot (e.g. hour), and is extended by proposing a robust clustering over multiple consecutive time-slots.

The paper is structured as follows: We first state the general BBU-RU clustering problem for the C-RAN architecture, and discuss standard C-RAN clustering performance metrics, in Section 2. We then present existing clustering methods from the literature in Section 3. In Section 4, we introduce our clustering solution based on the hyperbolic $\mathbb{H} 3$ distance and explain how it leads to the desired properties for the formed clusters; we also include its robust extension over multiple time-slots. In Section 5 we evaluate our algorithm using real data from Orange mobile France for two cities (Nantes, Lille) and compare its performance with existing approaches. Finally, Section 6 concludes our work.

\section{Problem statement}

In traditional RANs, each base station should be equipped with enough processing capacity in order to meet traffic demand at all times for the coverage area it is assigned to. However, since the traffic demand varies over different periods of the day, the processing capacity of base stations is 
not always used to its fullest. Chen et al., 2018 Moreover, different areas have different traffic patterns, for example business districts can have maximal charge during morning and afternoon hours in week days, while residential areas witness their rush hours during evenings and weekends. This means a certain BS can be overcharged during its peak hour while another BS in a different area can have minimal traffic at the same time, as shown in the example of Fig. 2a. In such scenarios, which occur very often in practice, the capacity of the low-charged BS is idling, whereas it could support traffic service for the overcharged one. Chen et al., 2018

In the C-RAN and vRAN architectures, BSs can be grouped together and cooperate by sharing common resources. In the example of Fig. 2a if the two BSs are mapped to the same BBU pool, their traffic is aggregated as shown in Fig. $2 \mathrm{~b}$, hence the capacity needed to serve both BSs simultaneously should at most cover the peak of their aggregated traffic (equal to 2.74), which is smaller than the sum of their individual peak traffic (equal to $1.75+1.61=3.36$ ). As a consequence, less processing units are necessary to be installed for the service of the group of these two BSs. What is more, during off-peak hours the pool resources are more intensively utilised (and do not idle).

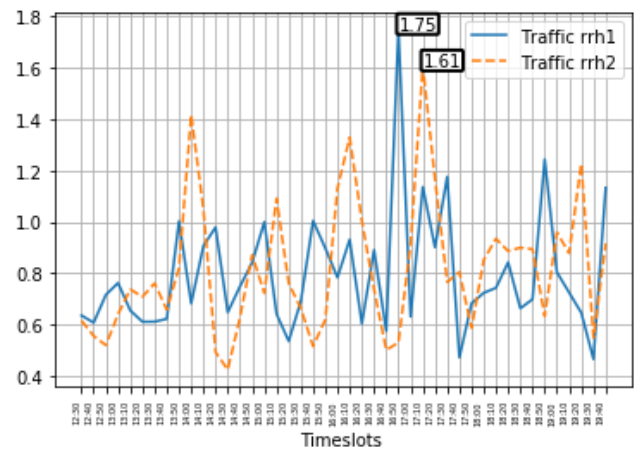

(a)

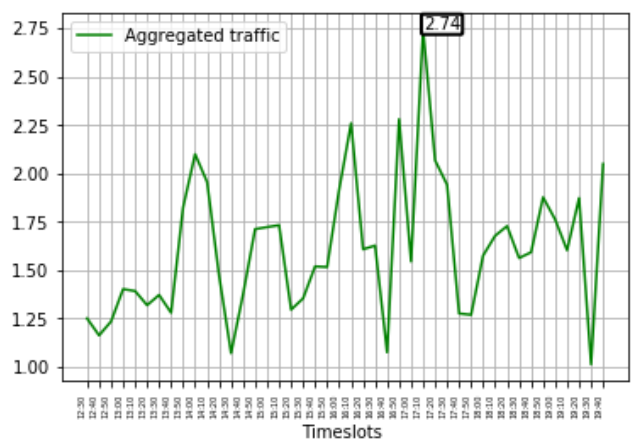

(b)

Fig 2: (a) Data traffic patterns of two RUs in Lille from 2019-06-10 16:10 to 2019-06-10 23:20 showing their traffic peak hour and volume (b) Their aggregated traffic.

The objective in this paper is to propose clustering (BS grouping) schemes that minimize the BBU resources needed to meet the traffic demand, under some distance limitations. These limitations have to do with the propagation time between RU and BBU, so that clustering very distant RUs to the same BBU-pool is not recommended due to unacceptable delays. Hence, the solution of a fully centralised cloud architecture with all RUs in a city served by the same BBU-pool is impossible, and we need to search for a semi-centralised solution that determines how many clusters should be optimally introduced per area (city), and which are their RU members.

To evaluate the quality of our proposed clustering solution, and compare it with existing ones, we will use two performance metrics, already introduced in. Chen et al., 2018 We use these because they elegantly quantify C-RAN clustering performance, and also in order to have coherence and a common vocabulary for the performance comparisons with other schemes. We will show that the clustering scheme proposed in this paper outperforms existing schemes, as evaluated from the metrics that follow. 


\subsection{Notation and Evaluation Metrics}

Let $z(n, t)$ be the traffic of station $n$ at time-slot $t$ for $n=1, \ldots, N$ and $t=1, \ldots, T$, where $N$ is the total number of stations in an area (city) and $T$ is the number of time-slots. Here, we will use as time-slot duration 1 hour, but other choice is possible depending on the available data. Requested traffic per station is normalised to 1 , so that the request per slot refers to the percentage of installed processing capacity utilised to satisfy traffic demand. We assume that all individual stations have the same amount of pre-installed processing capacity, so that $z(n)$ 's are comparable. After normalisation, we introduce a transformation of the traffic demand, called the remaining resources per station $n$ and time-slot $t$. This is equal to

$$
w(n, t)=1-z(n, t) .
$$

It quantifies the percentage of resources remaining idle per station per time-slot, and will be used in the proposed clustering algorithm.

Consider a partition $P$ of the set of $N$ stations into $K$ clusters, i.e $P=\left[C_{1}, \ldots C_{K}\right]$. Also, denote by $Z(C, t)$ the aggregated traffic at time $t$ of the stations grouped in cluster $\mathrm{C}$ i.e.,

$$
Z(C, t)=\sum_{n \in C} z(n, t)
$$

In the degenerate case of a cluster with a single station it holds, $Z(\{n\}, t)=z(n, t)$. Similarly, the aggregate remaining resources are defined as $W(C, t)=\operatorname{card}(C)-Z(C, t)$, where $\operatorname{card}(C)$ is the number of RUs forming the cluster.

Average-to-Peak Traffic Ratio (AtPTR) for cluster C. This is a measure of capacity utilisation per cluster. It is defined as the ratio of the average aggregate traffic request (i.e. average capacity used) at the BSs of the cluster for several time-slots, over the peak aggregate traffic.

$$
U(C)=\frac{\frac{1}{T} \sum_{t=1}^{T} Z(C, t)}{\max _{t} Z(C, t)}
$$

Obviously, $U(C) \in(0,1]$. When $U(C)$ is close to 1 the cluster makes good use of the pool resources over time, and resource idling is low. The AtPTR measure can be defined for just one station, i.e. $C=\{n\}$, in which case $U(n)=\frac{1}{T} \sum_{t=1}^{T} z(n, t) / \max _{t} z(n, t)$.

Metric I: Utilization for partition $P$. It is a measure of the improvement of the average AtPTR (i.e. capacity utilization per cluster) due to the partition $P$, compared to the average AtPTR over all single base stations in a traditional simple RAN,

$$
U \operatorname{til}(P)=\frac{\frac{1}{K} \sum_{k=1}^{K} U\left(C_{k}\right)}{\frac{1}{N} \sum_{n=1}^{N} U(n)} .
$$

It holds $\operatorname{Util}(P) \geq 1$. The equality holds when $K=N$, i.e. one cluster per BS. When traffic from several BSs in a cluster is aggregated, the pool resources are better utilized and the metric increases. The $\operatorname{Util}(P)$ is maximum for $K=1$. 
Metric II: Cost for partition $P$. It is a measure of the decrease in total installed BBU-pool resources in the clustered C-RAN, compared to the total BBU resources installed in individual base stations (simple RAN).

$$
\operatorname{Cost}(P)=\frac{\sum_{k=1}^{K} \max _{t} Z\left(C_{k}, t\right)}{\sum_{i=1}^{N} \max _{t} z(n, t)}
$$

where the maximum is taken over all time-slots $t=1, \ldots, T$. It holds $\operatorname{Cost}(P) \leq 1$. The equality holds again here when $K=N$. The $\operatorname{Cost}(P)$ is minimum when $K=1$.

The two performance metrics for a clustering $P$ quantify two different things. Metric I measures how much the utilisation of installed resources is improved by clustering $P$, whereas metric II measures the economies in installed resources due to clustering $P$, compared to the simple RAN standard scenario. Both are optimal for $K=1$. However, due to distance limitations, the solution to create a single global pool per city is not feasible.

\section{Clustering methods in the literature}

\subsection{Linear Programming}

Many research works in the literature try to formulate and solve the clustering problem as a Linear Program, see, Garcia-Saavedra et al., 2018 , Wang et al., 2016, da Silva Coelho et al., 2020 .u et al., 2020 Although this approach is definitely valid, high complexity issues may arise when the number of stations is citywide large, and especially when considering multi-period scheduling. Another issue is that most of these algorithms make arbitrary assumptions about the costs that will determine the clustering solution. The later can be strongly affected by small imprecisions in the measured costs (sensitivity of the solution). Finally, an important weak point is that the objective is maximised with binary (01) association and routing variables, that decide on which BBU-pool (or CU) to associate each RU with, without taking into account the relative geographic positions of the stations, and the potential interference these may introduce (or avoid) because of the specific partition as solution.

\section{$3.2 \mathrm{~K}$-means clustering}

An appropriate method that involves the $2 \mathrm{D}$-geometry and relative positions is by using data analysis. K-means is one of the most standard clustering algorithms, which can be used to partition the set of BSs of the city into $K$ disjoint clusters. Each BS is associated to a unique cluster, resulting in a partition $P$ that respects the following properties: ${ }^{\text {James et al., } 2013}$

1. $\bigcup_{k=1}^{K} C_{k}=\{1, \ldots, n\}$, i.e. the union of clusters includes all present BSs, where $C_{1}, C_{2}, \ldots, C_{K}$ are the $\mathrm{K}$ clusters.

2. $C_{k} \cap C_{k^{\prime}}=\varnothing$ for all $k \neq k^{\prime}$, i.e. clusters are disjoint sets.

K-means clustering is designed to minimize the within-cluster variance i.e regrouping the most similar BSs in the same cluster. This measure is defined as:

$$
\min _{C_{1}, C_{2}, \ldots C_{K}} \sum_{k=1}^{K} \operatorname{Var}\left(C_{k}\right),
$$


with $\operatorname{Var}\left(C_{k}\right)$ the within-cluster variance of the cluster $\mathrm{k}$,

$$
\operatorname{Var}\left(C_{k}\right)=\frac{1}{\operatorname{card}\left(C_{k}\right)} \sum_{i \in C_{k}} d^{2}\left(i, m_{k}\right) .
$$

The $m_{k}$ is the centroid of the cluster $k$, and in euclidean space it is the point having as coordinates the average values of all RUs in the cluster. In 2D it is defined as $m_{k}=\left(\bar{x}_{k}, \bar{y}_{k}\right)$.

The $d^{2}(i, j)$ is the square of the distance between station $i$ and the cluster centroid $m_{k}$. If we assume that the RUs are embedded on the 2D euclidean space, the distance $d_{E 2}\left(x_{i}, y_{i}, x_{j}, y_{j}\right)=$ $\sqrt{\left(x_{j}-x_{i}\right)^{2}+\left(y_{j}-y_{i}\right)^{2}}$ can be used, which makes a lot of sense in the C-RAN and vRAN problem, where base stations are positioned on the 2D plane; it can be favorable to group stations together that are closer to each other, thus avoiding inter-clustering hand-overs and bringing all RUs closer to the BBU-pool, positioned at the cluster centroid. Note, however, that this approach makes use only of the two geographical coordinates $\left(x_{n}, y_{n}\right)$ per BS $n=1, \ldots, N$, and does not include the traffic demand feature.

A naive extension to the $3 \mathrm{D}$, is to include all dimensions $(x, y, z)$, (or $(x, y, w)$ for the resources), where the variables $z$ and $w$ refer to a specific time-slot $t$. As we will show later, the euclidean 3D distance $(E 3)$ is not the best choice, because the clusters formed do not exhibit the desired properties. The pseudo-algorithm for 2D euclidean K-means is described as follows (it extends to $3 \mathrm{D}$ or higher dimensions in a straightforward way):

Algorithm: Euclidean K-means Clustering in 2D

1. Init: Fix a number $K$ of clusters and randomly assign each RU to one of the $\mathrm{K}$ clusters.

2. Iterate until the cluster assignments stop changing:

(a) Compute the centroid of each cluster. The $k$ th cluster centroid is the $m_{k}=\left(\bar{x}_{k}, \bar{y}_{k}\right)$ average position of the RUs in the $k$ th cluster.

(b) Assign each BS to the cluster whose centroid is closest according to the 2D-Euclidean distance.

\subsection{Distance-Constrained Clustering Algorithm}

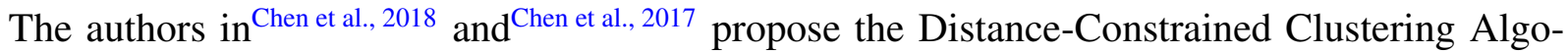
rithm (DCCA) for RU clustering. This algorithm takes into consideration not only the position, but also the traffic-demand and its temporal evolution through the day, while respecting some distance constraint. The method introduces an entropy-based weight to evaluate the complementarity of traffic between pairs of RUs over a determined time-interval. RUs are placed as nodes of a weighted-graph, whose edges can exist or not and allow clusters to form. Each link has a binary activation variable controlled by the algorithm; when this becomes 1 , an edge appears between the two RUs, which can now share their resources and belong to the same cluster. The authors further introduce a distance constraint, so that only RUs within some distance from each other are allowed to collaborate. 
Although this approach sets the problem in a correct framework, it is sub-optimal because the complementarity of a large group of RUs is calculated as the sum of complementarities between pairs of these RUs. This is of course not correct: in a scenario of three stations, both $R U_{2}$ and $R U_{3}$ may have large traffic demand, which renders them complementary in traffic with $R U_{1}$ which has low charge; but placing them all three in the same cluster should normally be avoided, because $R U_{2}$ and $R U_{3}$ are not mutually complementary. The pairwise comparison is thus suboptimal, and other approaches which consider the joint-complementarity for the whole set of RUs in a cluster should be investigated.

\section{Hyperbolic K-means}

In this section our novel suggestion for RAN clustering is introduced.

\subsection{Traffic-aware RU clusters}

The main challenge is how to incorporate the traffic demand inside the clustering algorithm, additionally to the positional features. To achieve this, we first present three qualitative criteria that should be satisfied by any traffic-aware clustering:

1. When two RUs have complementary traffic (or remaining resources), i.e, one has high traffic load (resp. low resources) and the other low traffic load (resp. high resources), they should be grouped together, in order to cooperate, within certain geographical limitation of distance.

2. When two base stations both have low traffic volume (resp. high resources), it is irrelevant whether they cooperate or not, because of low benefit.

3. When two base stations both have high traffic volume (resp. low resources), their cooperation leads to no benefit and they should not be clustered together. Ideally, highly loaded stations should be distributed evenly among several clusters.

We saw in Section 3.2 that the K-means approach for 2D has the advantage to summarise well the information from all members of the cluster at its centroid, with coordinates being the averages of the $x$ and $y$ coordinate of all RUs in the cluster. Hence, it is by construction a better approach than the DCCA. Furthermore, the method generates clusters that are compact, thus avoiding to include RUs that spread over large distances from the centroid of each cluster. It has two drawbacks, however: the vanilla $\mathrm{K}$-means in 2D does not include the traffic dimension; it also generates one partition per time-slot, hence does not easily generalise to longer time-intervals.

A naive way to incorporate the resource dimension $w$ (or the traffic dimension $z$ ) in the Kmeans, as additional feature to the position $(x, y)$ of the RU, is to consider the 3D euclidean metric space and its corresponding euclidean distance. From now on we will use $w$ as the third feature. The $3 \mathrm{D}$ euclidean distance between $R U_{i}=\left(x_{i}, y_{i}, w_{i}\right)$ and $R U_{j}=\left(x_{j}, y_{j}, w_{j}\right)$ is

$$
d_{E 3}\left(x_{i}, y_{i}, w_{i}, x_{j}, y_{j}, w_{j}\right)=\sqrt{\left(x_{i}-x_{j}\right)^{2}+\left(y_{i}-y_{j}\right)^{2}+\left(w_{i}-w_{j}\right)^{2}} .
$$

The above expression can be re-written as $\sqrt{d_{E 2}^{2}\left(x_{i}, y_{i}, x_{j}, y_{j}\right)+\left(w_{i}-w_{j}\right)^{2}}$, which relates the 3D with the 2D euclidean distance, and the difference of the remaining resources. This specific choice, although natural, does not achieve the desired effect, since it treats the traffic and position in a 
homogeneous way, although the units and range of geography and traffic are completely different. The $(x, y)$ is positioned in the real 2D-space $((-\infty,+\infty) \times(-\infty,+\infty))$ and the features $x, y$ are measured in meters, or kilometers, whereas the resource dimension is in BBU units, so that $w \in[0,1]$ (the BBU capacity is normalised to 1 ). Another issue with the choice of 3D euclidean distance, is that two RUs have minimum distance when $w_{i}=w_{j}$, i.e. when both stations have the same remaining resources, irrespective of whether this quantity is high or low. Moreover, given a fixed difference $\left(w_{i}-w_{j}\right)^{2}$, the square of the 3D-distance is proportional to the square of the 2D-distance; as a result, RUs in a small geographical distance from each other are favored to collaborate, whereas large 2D distance prohibits any collaboration.

Given the above observations, the 3D euclidean distance is not the best candidate to apply Kmeans, when aiming to achieve a clustering with balanced load (i.e. inter-cluster complementarity), and we need to look for other candidates, that treat the traffic dimension in a way different than the RU position.

\subsection{Hyperbolic Distance}

An interesting idea is to embed the 3D features of each RU $\left(x_{n}, y_{n}, w_{n}\right)$ into a hyperbolic space instead, and use the distance induced by the Poincare metric. The idea of embedding complex data into hyperbolic space is recent, but is gaining momentum. It has already been successfully applied for the analysis of complex networks in, Krioukov et al., 2010 as well for developping new methods to learn symbolic data which exhibit hierarchy and similarity from Facebook's research team in. Nickel and Kiela, 2017 These highly cited works show that for problems of clustering and community detection, the hyperbolic space is more appropriate than the Euclidean. Very recently, the authors in $^{\text {Hajri et al., } 2019}$ have formulated the K-means clustering algorithm in the hyperbolic setting, where they use the Poincaré ball model [Loustau, 2020, Chapter 8.1]. Further contributions include the introduction of hyperbolic space algorithms for community detection. Gerald et al., 2020

In the majority of the aforementioned methods data is embedded into the Poincaré ball model, which in $3 \mathrm{D}$ is the manifold $\mathbb{B}^{3}=\left\{(x, y, w) \in \mathbb{R}^{3}:\|(x, y, w)\|<1\right\}$. In our case, the positional features $(x, y) \in \mathbb{R}^{2}$ are real numbers, but the feature of remaining resources is in fact real positive $w \in \mathbb{R}_{+}$. So, we choose to embed the data on the Poincaré half-plane model instead [Loustau, 2020, Chapter 8.2], which in 3D is the manifold $\hat{\mathbb{B}}^{3}=\left\{(x, y) \in \mathbb{R}^{2}, w \in \mathbb{R}_{+}:\|(x, y, w)\|<1\right\}$, with induced distance between $R U_{i}=\left(x_{i}, y_{i}, w_{i}\right)$ and $R U_{j}=\left(x_{j}, y_{j}, w_{j}\right)$

$$
d_{H 3}\left(x_{i}, y_{i}, w_{i}, x_{j}, y_{j}, w_{j}\right)=\operatorname{arccosh}\left(1+\frac{d_{E 3}^{2}\left(x_{i}, y_{i}, w_{i}, x_{j}, y_{j}, w_{j}\right)}{2 w_{i} w_{j}}\right)
$$

where $\operatorname{arccosh}(s)=\ln \left(s+\sqrt{s^{2}+1}\right)$, an increasing function of the argument $s$. This specific hyperbolic distance (we refer to it from now on in short as $H 3$ ) incorporates both the Euclidean 2D distance (involving the positional pair $(x, y)$ ), as well as the impact of resource imbalance $w_{i}$ and $w_{j}$, Decreusefond et al., 2012. Álvarez-Corrales et al., 2017 It is clear from (9) that the hyperbolic distance is monotone increasing in $d_{E 3}$. However, the third resource dimension $w$ plays a special role as it divides the $d_{E 3}$. As a consequence, when the absolute available resources $w_{i}, w_{j}$ are small, their product is small, and the distance between $R U_{i}$ and $R U_{j}$ is large. This is important because it generates the tendency to place RUs with low resources (high load) in separate clusters. 
With a bit of calculus, the new distance takes another interesting form

$$
d_{H 3}\left(x_{i}, y_{i}, w_{i}, x_{j}, y_{j}, w_{j}\right)=\operatorname{arccosh}\left(\gamma \frac{d_{E 2}^{2}\left(x_{i}, y_{i}, x_{j}, y_{j}\right)}{2 w_{i} w_{j}}+\frac{1}{2}\left(\frac{w_{i}}{w_{j}}+\frac{w_{j}}{w_{i}}\right)\right) .
$$

Note the new parameter $\gamma>0$ inserted above. When $\gamma=1$, the expression in (10) is equal to (9). The reason why $\gamma$ is introduced is very important. We see from above that the argument of arccosh has two summands. The first is described by the $2 \mathrm{D}$-euclidean distance with $(x, y)$ and the product of the resource feature $w$, whereas the second depends only on the resource feature $w$. The second summand quantifies the resource imbalance between $w_{i}$ and $w_{j}$. It is 0 when $w_{i}=w_{j}$ and positive when $\left\|w_{i}-w_{j}\right\|>0$. In fact, it is symmetric regarding an imbalance towards $w_{i}$ or $w_{j}$. The hyperbolic distance depends on these two summands, and trades-off between geographical distance and resource imbalance. The issue is however, that 2D-distance and resources do not have the same units! If the $d_{E 2}$ is measured in meters, the geographical distance between two RUs can be of the order of thousands, and can dominate the summation. If the $d_{E 2}$ is measured in kilometers instead, the resource balance summand will dominate, thus giving more emphasis on whether two RUs have the same amount of resources or not. Although the units depend on the convention we make, each choice will lead to a different clustering result. This is why, we choose for now to leave $\gamma$ as a tunable parameter (or just $\gamma=1$ ), and investigate its influence in the evaluation Section 5 . We can potentially choose an appropriate $\gamma$ function that maximises our metrics.

\subsection{H3 distance properties}

To get a better understanding on the properties of the hyperbolic distance $H 3$ and how this compares to the 3D-euclidean $E 3$, we introduce here a transformation of the pair of resource variables $\left(w_{i}, w_{j}\right)$. The imbalance of the two variables will be described by their ratio $\rho>0$. Furthermore, let us fix their sum to $C>0$ resource units. Then, the tuple $\left(w_{i}, w_{j}\right)$ can be uniquely expressed as a function of $(\rho, C)$ and vice versa,

$$
\left.\left.\begin{array}{l}
C=w_{i}+w_{j} \\
\rho=w_{i} / w_{j}
\end{array}\right\} \Leftrightarrow \begin{array}{l}
w_{i}=\rho \cdot C /(1+\rho) \\
w_{j}=C /(1+\rho)
\end{array}\right\}
$$

Let us also denote the $2 \mathrm{D}$ euclidean distance of the RU positions by $\delta=d_{E 2}\left(x_{i}, y_{i}, x_{j}, y_{j}\right)$. The hyperbolic distance $H 3$ in (10) can be expressed as a function of $(\delta, \rho, C)$ (we assume $\gamma=1$ w.l.o.g.)

$$
d_{H 3}(\delta, \rho, C)=\operatorname{arccosh}\left(\frac{\delta^{2}}{2 \rho \frac{C^{2}}{(1+\rho)^{2}}}+\frac{1}{2}\left(\rho+\frac{1}{\rho}\right)\right) .
$$

In a similar way, the $3 \mathrm{D}$ euclidean distance $(E 3)$ can also be expressed by the same arguments

$$
d_{E 3}(\delta, \rho, C)=\sqrt{\delta^{2}+C^{2} \frac{(1-\rho)^{2}}{(1+\rho)^{2}}} .
$$

We already observe a difference in the expressions (13) and (12). The 3D-euclidean distance is increasing in the sum of resources $C$, whereas the hyperbolic distance is decreasing (remember $\operatorname{arccosh}(s)$ is an increasing function of $s$ ). Hence, the 3D-euclidean distance will perceive two RUs 
having low remaining resources as being close to each other, something which leads to inefficient clustering for C-RANs. To further understand why the hyperbolic distance is more appropriate in our scenario, we will keep two parameters fix in the above expressions and plot its response when increasing the third parameter.

- Fix $C$ and $\rho$, vary the 2D-euclidean distance $\delta$. The plot is in Fig. 3 for both distances. We observe that the 3D-euclidean increases proportionally to the 2D-euclidean with unit slope. The hyperbolic distance, on the other hand, deforms the influence of the 2D-distance. The hyperbolic is a concave function of the 2D-euclidean and increases with a slope less than 1 . As a result, RUs that are distant in the 2D-sense are perceived closer through the hyperbolic lens.

- Fix $\delta$ and $\rho$, vary the sum of resources $C$. The plot is in Fig. 4 for both distances and we can see the difference in their behaviour. In contrast to the 3D-euclidean, the hyperbolic is monotone decreasing in $C$, hence two RUs with small remaining resource sum are perceived far from each other and should not be placed in the same cluster. What is also striking is that the downward slope of $H 3$ is very large in the whole range of $C$ values (diminishing for very large $C$ ), which indicates that this distance is very sensitive to a small change in the resource sum. This comes in striking contrast with $E 3$ which is increasing in $C$ with a very small slope, hence quite insensitive.

- Fix $\delta$ and $C$, vary the imbalance ratio $\rho=w_{i} / w_{j}$. The plot is in Fig. 4 for both distances. The range of values is chosen in $[0.001,1000]$, so for $\rho<1$ the resources are imbalanced in favor of $w_{j}$ and for $\rho>1$ it is the other way round. We see that both the hyperbolic and the 3D-euclidean are symmetric, with axis of symmetry $\rho=1$. But the $H 3$ is much more sensitive to resource imbalance than E3. As the plot illustrates, $H 3$ increases fast due to imbalance, in a symmetric fashion around $\rho=1$, with a minimum when the two RUs are completely balanced, i.e. have the same number of remaining resources.

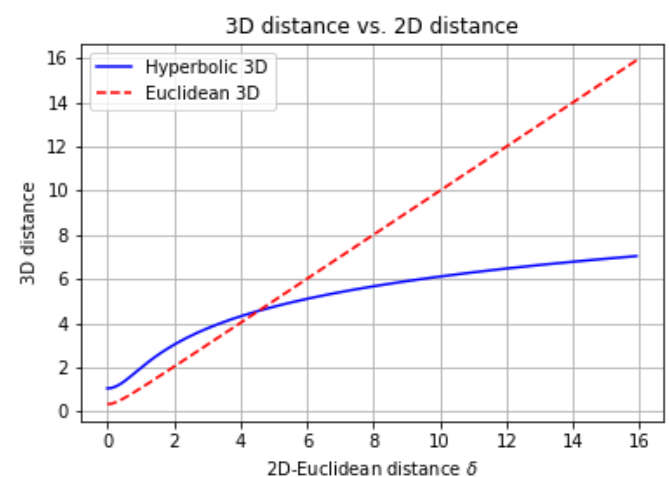

Fig 3: Hyperbolic and euclidean 3D-distance when varying the 2D-euclidean distance $\delta$, for fixed $C=1$ and $\rho=2$.

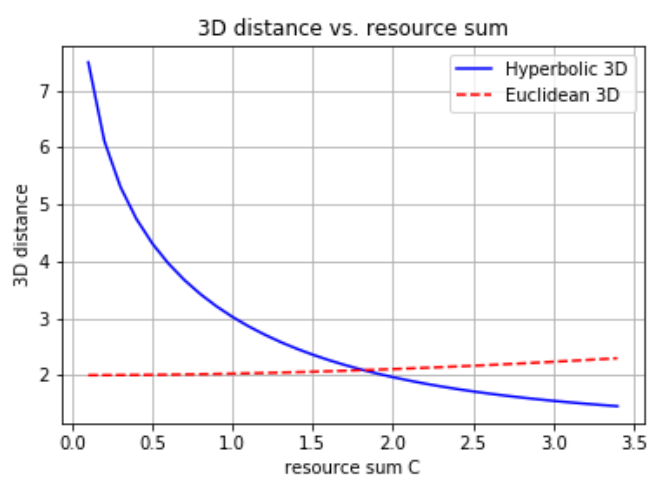

Fig 4: Hyperbolic and euclidean 3D-distance when varying the resource sum $C$, for fixed $\delta=$ 2 and $\rho=2$. 


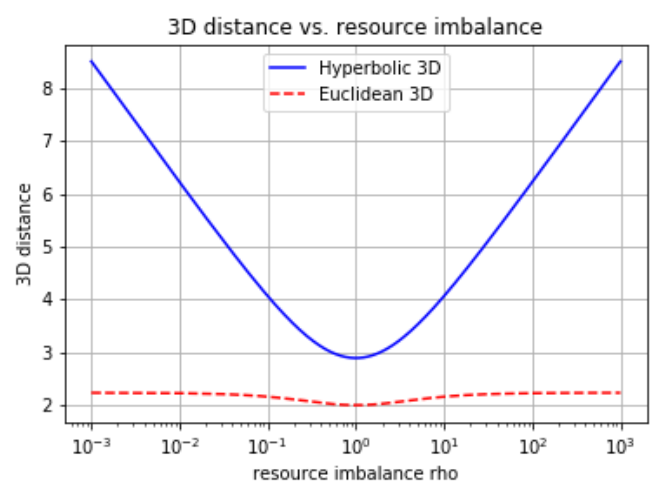

Fig 5: Hyperbolic and euclidean 3D-distance when varying the imbalance ratio $\rho$, for fixed $C=1$ and $\delta=2$.

\subsection{Hyperbolic K-means algorithm for RU clustering}

We are now ready to incorporate the $H 3$ distance for K-means clustering in C-RAN. As observed in Fig. 5, the 3D-hyperbolic distance (and also the euclidean) between two points $\left(x_{i}, y_{i}, w_{i}\right)$ and $\left(x_{j}, y_{j}, w_{j}\right)$ is minimal when the resource ratio $\rho=1$, meaning that the resources are balanced $w_{i}=w_{j}$. This property can be exploited for clustering, by introducing the centroid of each cluster $C_{k}$. Let us define the centroid $m_{k}=\left(x_{k}, y_{k}, w_{k}\right)$ coordinates, again as the averages

$$
\left(x_{k}, y_{k}, w_{k}\right)=\frac{1}{\operatorname{card}\left(C_{k}\right)} \sum_{j \in C_{k}}\left(x_{j}, y_{j}, w_{j}\right) .
$$

In every update loop, each $R U_{n}, n=1, \ldots, N$ will be associated to the cluster with minimum hyperbolic distance $d_{H 3}\left(n, m_{k}\right)$ between the specific $R U_{n}$ and the centroid. Forgetting for a moment the effect of the positional pair dimensions $(x, y)$ (the 2D-euclidean distance), such rule will try to associate stations to clusters in a way that changes as little as possible the average available resources $w_{k}$. A further consequence is that the resulting clusters will have balanced average traffic in a fair way (see Example below).

In this case, the within-cluster variance of a given cluster $C_{k}$ having as centroid $m_{k}\left(x_{k}, y_{k}, w_{k}\right)$ is defined as follows

$$
\operatorname{Var}_{H 3}\left(C_{k}\right)=\frac{1}{\operatorname{card}\left(C_{k}\right)} \sum_{i \in C_{k}} d_{H 3}^{2}\left(i, m_{k}\right),
$$

and the global distortion is the sum of variances over all clusters

$$
V_{H 3}(P)=\sum_{k=1}^{K} \operatorname{Var}_{H 3}\left(C_{k}\right) .
$$

Algorithm 3: Hyperbolic K-means Clustering in 3D 
1. Init: Fix a number $K$ of clusters and chose randomly the initial cluster centroids $\left(x_{k}, y_{k}, w_{k}\right)$. Also, fix the tolerated distortion error $\epsilon>0$.

2. Assign each RU to the cluster whose centroid is the closest according to the hyperbolic distance $H 3$.

3. Calculate the initial global distortion $V_{H 3}(0)$.

4. Iterate while the difference between the global distortions of two consecutive iterations is larger than $\epsilon:\left|V_{H 3}(\tau)-V_{H 3}(\tau-1)\right|>\epsilon$

(a) Compute the centroid of each cluster by (14).

(b) Assign each observation to the cluster whose centroid is closest according to the distance $H 3$.

(c) calculate the global distortion $V_{H 3}$.

Implementation: In Step 1 (initialisation), we place the $K$ initial centroids at the positions of $K$ RUs randomly among the present $N$ RUs. This choice aims to guarantee that no cluster will start empty, and in practice leads to $K$ non-empty clusters after convergence. Since the centroids are initialised randomly, and since the $K$-means algorithm is known not to converge to a global optimum, we repeat the algo. 3 several times (use a counter count) and then we pick the solution with maximum performance, among the count available ones.

Example: To see how the hyperbolic K-means works, with the resulting fairness among clusters, we make a thought experiment. Suppose $R U_{1}=(-\delta, 0, \alpha w), R U_{2}=(0,0, w)$ and $R U_{3}=$ $(+\delta, 0, w / \alpha)$ are co-linear in the $\mathrm{x}$-axis, as their coordinates indicate. For now, let $\alpha>0$. The $R U_{2}$ has the same 2D-euclidean distance $\delta$ from both $R U_{1}$ and $R U_{3}$. Suppose we want to find $K=2$ clusters. Will $R U_{2}$ be clustered with $R U_{1}$ or with $R U_{3}$ ?

The cluster centroids are initialised as $m_{1}=R U_{1}$ and $m_{3}=R U_{3}$. The association of $R U_{2}$ is with $R U_{1}$ if $d_{H 3}\left(R U_{2}, R U_{1}\right)<d_{H 3}\left(R U_{2}, R U_{3}\right)$. Using the expression from (10) with $\gamma=1$, we see that this inequality is valid when $\alpha>1$. So, when $\alpha>1$, the clustering $C_{1}=\left\{R U_{1}, R U_{2}\right\}$ and $C_{2}=\left\{R U_{3}\right\}$ will update the centroid coordinates as $m_{1}^{\prime}=(-\delta / 2,0, w(1+\alpha) / 2)$ and $m_{2}^{\prime}=$ $(+\delta, 0, w / \alpha)$. As a result, the difference of average resources between cluster $C_{1}$ and $C_{2}$ equals to $\Delta w=\|w(1+\alpha) / 2-w / \alpha\|$ is smaller (more fair) compared to the situation when the $R U_{2}$ would be clustered with $R U_{3}$, in which case $\tilde{\Delta} w=\|w \alpha-w(1+1 / \alpha) / 2\|>\Delta w$.

The association will not change after the update of centroids, because for $\alpha>1$ it can be verified that it still holds $d_{H 3}\left(R U_{2}, m_{1}^{\prime}\right)<d_{H 3}\left(R U_{2}, m_{2}^{\prime}\right)$.

\subsection{Robustness}

Up to this point, we have considered a single traffic value per station per time-slot $t$. As explained at the beginning, the traffic varies over time, and suppose we have several measurements per station through the day. We thus need to obtain a robust clustering over multiple consecutive time-slots.

As a first naive idea, we can consider averaging the traffic per RU over all the $T>1$ timeslots, and then perform our Hyperbolic K-means using average data as 3rd dimension. This idea 
however simple, is not appropriate, because it flattens the trace and loses all information over RU complementarity in different time-slots. Imagine for example that in a certain slot two stations $i$ and $j$ are complementary with $w_{i}>w_{j}$, whereas a couple of slots later the situation is inverted with $w_{i}<w_{j}$; the two stations still are complementary as pair. If we average over these two time slots, the complementarity is lost, because the two RUs have similar values of average traffic. To avoid such loss of information, we introduce Algorithm 4, which extends our Hyperbolic K-means over multiple time-slots.

Let $T$ be the number of consecutive time-slots to consider. For each time slot $t$, we apply Algorithm 3 using the resource values $w(n, t)$ measured per RU $n$ and for the specific time slot $t$. Obviously the RU 2D-euclidean positions $\left(x_{n}, y_{n}\right)$ do not change over time, but the cluster centroids can change because of the third dimension. Let $P(t)=\left\{C_{k}(t), k=1, \ldots, K\right\}$ be the obtained clustering per time slot $t$, and let $\varphi(t)=\left\{m_{k}(t), k=1, \ldots, K\right\}$ be the set of cluster centroids of $P(t)$. It is important to note that every partition has exactly $K$ centroids (in the degenerate case two or more centroids may be ovelapping).

The robust algorithm works as follows: The centroids of the first time-slot $\varphi(1)$ are considered as reference. For each centroid $m_{k}(1)$ in the the set, find the $(T-1)$ centroids closest to it from the centroid sets of the next $T-1$ partitions $\varphi(t)$. Then calculate their average on each of the three dimensions $(x, y, w)$. That is to say, if $T=2$ and $m_{k}(1)=\left(x_{k}(1), y_{k}(1), w_{k}(1)\right)$ is the $k$-th centroid obtained at time-slot $t=1$, then we search for $m_{k^{\prime}}(2)=\left(x_{k^{\prime}}(2), y_{k^{\prime}}(2), w_{k^{\prime}}(2)\right)$, the closest centroid to $m_{k^{\prime}}(2)$ from an execution at $t=1$. The resulting robust centroid is the average of each dimension of these two centroids $m_{k}(1)$ and $m_{k^{\prime}}(2)$. We finally remap the RRHs according to the new centroids using the $H 3$ distance.

Algorithm 4: Robust Hyperbolic K-means for RU clustering

1. For each time slot $t=1, \ldots, T$ :

(a) Execute algorithm 3 for all $t=1, \ldots, T$ and obtain the set of centroids $\varphi(t)=$ $\left[m_{1}(t), \ldots, m_{K}(t)\right]$.

2. Given the first set of centroids $\varphi(1)$, for each $m_{k}(1) \in \varphi(1), k=1, \ldots, K$ :

(a) Get the closest centroid to $m_{k}(1)$ from each partition $\varphi(t), t=1, \ldots, T$.

(b) Compute the average $k$-th centroid over all time realisations.

3. Assign each RU to the clusters according to their $H 3$ distance to the new average (robust) centroids. 


\section{Evaluation}

In this section, we use real-world mobile data provided by Orange, France for two cities (Lille and Nantes). First, we study the effect of parameters on the performance of the Hyperbolic Kmeans. Then, we apply the metrics mentioned in Section 2.1 to compare the utilization and cost of our hyperbolic algorithm 3 (and its robust version 4) against other existing solutions: (i) the K-means clustering using the 2D-euclidean distance (considering just the RU position without the traffic / resources), (ii) the 3D-euclidean distance (with remaining resources as third feature), (iii) the DCCA clustering.

\subsection{Datasets description}

We use for our evaluation mobile data provided by Orange, France that contains four months of traffic data from 2019-03-19 to 2019-06-16 for Lille and from 2019-03-16 to 2019-06-16 for Nantes. In addition, we use a dataset of the RUs positions for each city.

Lille and Nantes contain respectively $N=88$ and $N=97$ RUs. An RU position consists of its geographical coordinates $\left(x_{n}, y_{n}\right)$ in the Lambert II Carto projection system. Fig. 6 shows how the areas of the two cities are partitioned in a Voronoi diagram.

The traffic dataset contains per antenna, its traffic in Bytes-Up and Bytes-Down considered in 10-minute-long time-slots. The Bytes-Down traffic volume is more important than Bytes-Up, as we can see in Fig. 7, and thus is more representative. Therefore, we will only use the Bytes-Down traffic for evaluation.

Table 1 summarizes the dataset description.

Table 1: Data Description

\begin{tabular}{|c|c|c|}
\hline Dataset & Lille & Nantes \\
\hline Number of antennas & 1394 & 1413 \\
\hline Number of RU positions & 88 & 97 \\
\hline Data collection period & 2019-03-19 to 2019-06-16 & 2019-03-16 to 2019-06-16 \\
\hline Time-slot duration & 10 -minute & 10 -minute \\
\hline Maximal traffic Bytes-Up & $7.2510^{9}$ & $3.0410^{9}$ \\
\hline Minimal traffic Bytes-Up & 0.0 & 0.0 \\
\hline Maximal traffic Bytes-down & $15.8210^{9}$ & $18.1510^{9}$ \\
\hline Minimal traffic Bytes-down & 0.0 & 0.0 \\
\hline
\end{tabular}



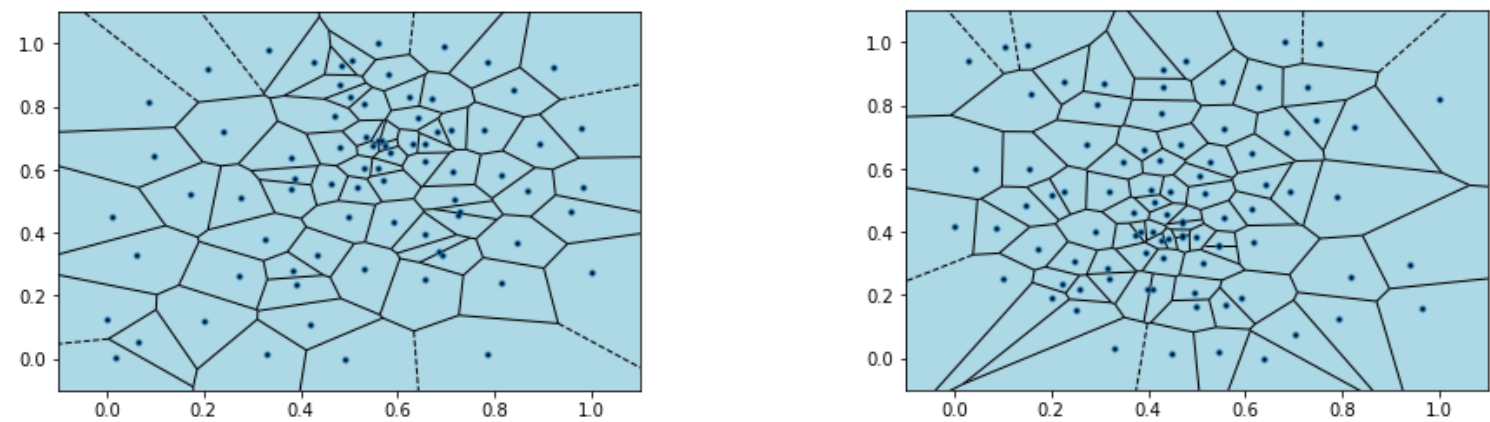

Fig 6: Voronoi partitions of Lille (left) and Nantes (right)
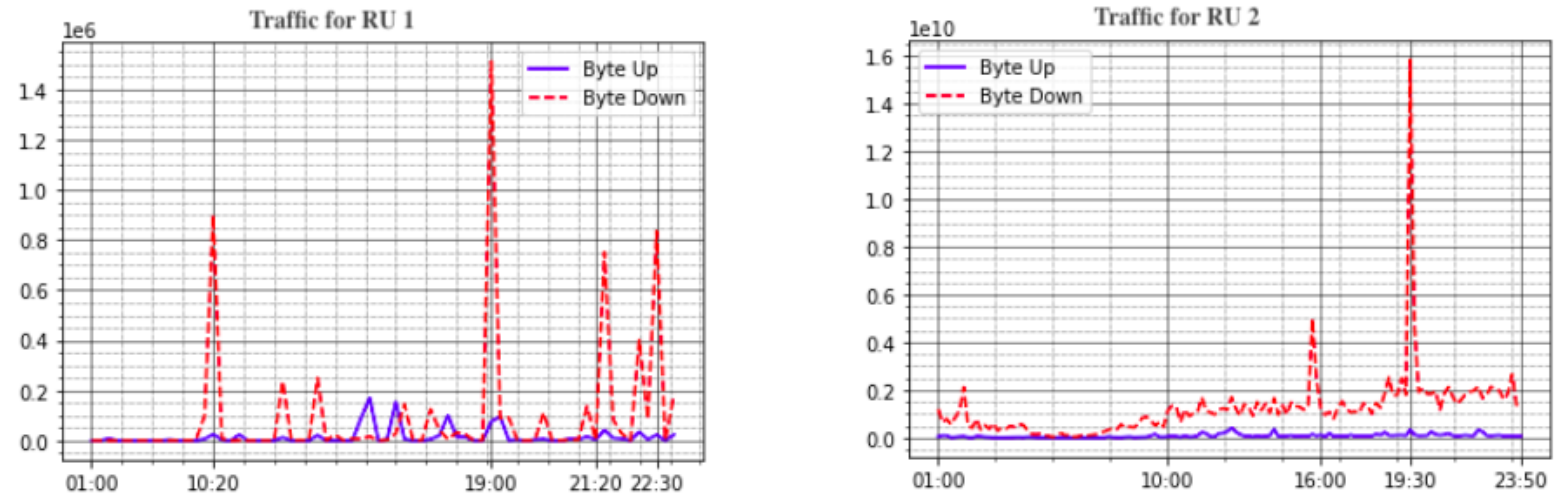

Fig 7: Example of Bytes-Up and Bytes-Down patterns of two RUs

\subsection{Evaluation Plan}

In order to evaluate the algorithms, we construct a typical week traffic profile according to the Base Stations Traffic Profile Generation method proposed in. Chen et al., 2017

Given our traffic dataset, for each RU, we aggregate and average the traffic of each timeslot of each day of the week over the four months of traffic data to construct the typical traffic of the week, as shown in Fig. 8.

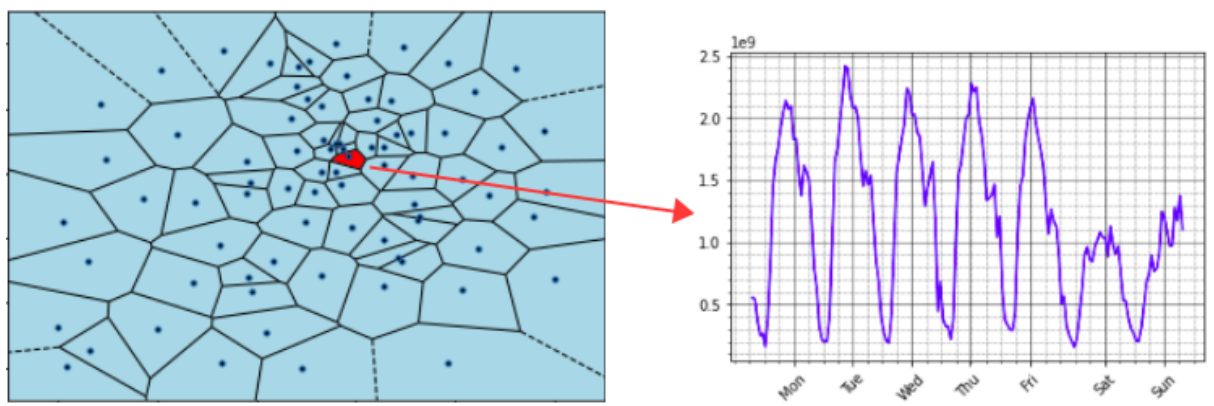

Fig 8: An example of the traffic pattern from a chosen RU. 
The traffic and positions are normalized for evaluation by the following method: for each $\left(x_{n}, y_{n}, z_{n}\right)$ from the dataset, where $x_{n}$ is the first geographical coordinate, $y_{n}$ is the second geographical coordinate and $z_{n}$ is the traffic, the corresponding normalized values are :

$$
\left(\tilde{x}_{n}=\frac{x_{n}-\min \mathcal{X}}{\max \mathcal{X}-\min \mathcal{X}}, \tilde{y}_{n}=\frac{y_{n}-\min \mathcal{Y}}{\max \mathcal{Y}-\min \mathcal{Y}}, \tilde{z}_{n}=\frac{z_{n}-\min \mathcal{Z}}{\max \mathcal{Z}-\min \mathcal{Z}}\right)
$$

In the above $(\mathcal{X}, \mathcal{Y})$ is the dataset over the positions, and $\mathcal{Z}$ is the dataset of traffic for the considered period of clustering. The normalised values $\left(\tilde{x}_{n}, \tilde{y}_{n}, \tilde{z}_{n}\right)$ will be used in the evaluation metrics of utilisation (4) and cost (5). Furthermore, we define the normalised resources $\tilde{w}_{n}=1-\tilde{z}_{n}$ for all stations and time-slots, which will be used in the hyperbolic K-means algorithms.

\subsection{Parameter Effect Study}

We start by evaluating the effect of certain design parameters in the hyperbolic K-means: the $\gamma$ scaling parameter, the number $K$ of clusters, and the duration $T$ of consecutive time slots. (For the first two parameters we cluster for a single time-slot $t$ ). We evaluate the cost and utilization for different values of these parameters and determine an optimal set that we use later for comparison against the existing clustering algorithms.

\subsubsection{The scaling parameter $\gamma$}

The Hyperbolic $H 3$ distance used in the K-means has been defined in (10). In this expression, the scaling parameter $\gamma$ was introduced to determine through scaling, whether the focus should be on the RU positions ( $\gamma$ large) or their traffic ( $\gamma$ small). For very large values of $\gamma$, only the first summand in the argument is important, putting the focus almost entirely on the RUs positions. In this case the behaviour of Hyperbolic K-means should be similar to that of 2D-Euclidean K-means.

We run the Hyperbolic K-means (algo. 3) on the normalized traffic data for a number of clusters $K=6$ and various values of $\gamma \in(0,20)$ to evaluate its impact on the utilization and cost of the obtained partitions. We also run for comparison the two Euclidean K-means algorithms (2D- and 3D-), which do not depend on $\gamma$, for the same number of clusters $K=6$.

Take-away 1: We observe in Fig. 9a and Fig. $9 \mathrm{~b}$ that the hyperbolic K-means shows significant improvement in utilization performance compared to the euclidean K-means algorithms for values of $\gamma \leq 7$. For higher values of $\gamma$ all three algorithms tend to converge to equal utilization performance. For small values of $\gamma$, we get better cost and utilisation by neglecting in practice the RU positions. This allows for clusters to be formed that have wide geographical spread (diameter) but might not respect some design distance constraints related to the fiber length, as Fig. 10 indicates. In this figure we evaluate the diameter of a partition. This is defined here as the max distance between two RUs in the same cluster (for all clusters in the partition)

$$
\operatorname{Diam}(P)=\max _{k} \max _{i, j \in C_{k}} d_{E 2}^{2}(i, j)
$$

In the above, $d_{E 2}^{2}(i, j)$ applies the 2D-euclidean distance between the positions of $R U_{i}$ and $R U_{j}$. The figure shows that for $\gamma<0.5$ the cluster diameter increases considerably. 


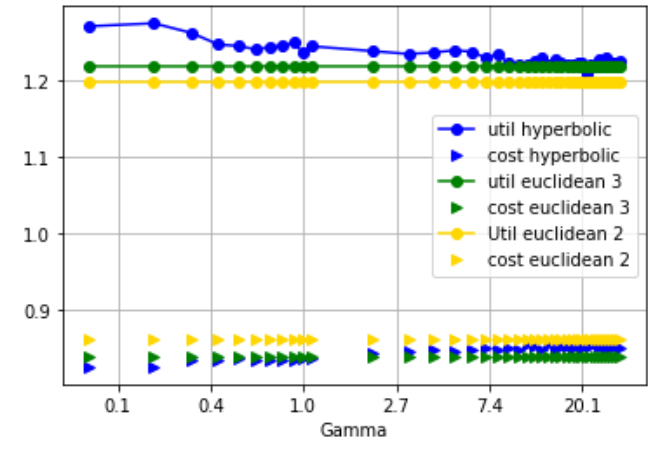

(a) Lille

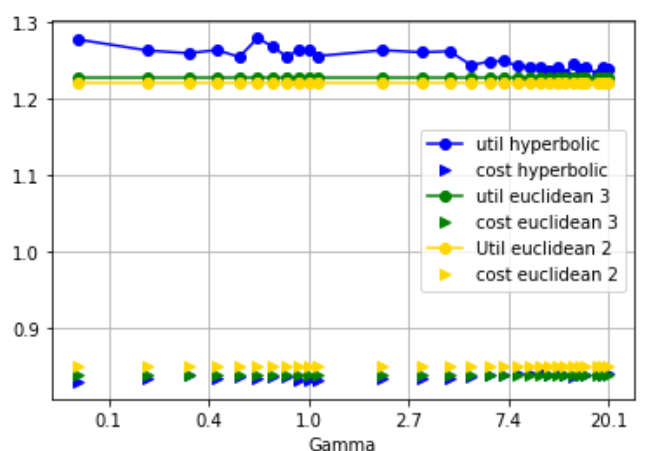

(b) Nantes

Fig 9: Utilization and Cost of the k-means clustering using Hyperbolic, Euclidean 2D and Euclidean 3D distances according to $\gamma$.

Since the main role of $\gamma$ is to appropriately scale the 2D-distance and the resource dimension to achieve homogeneity in the $H 3$ expression, we choose for the rest of the evaluation tests $\gamma=1$ since our data-sets are normalized in $[0,1]$ for both the traffic and the RU positions.

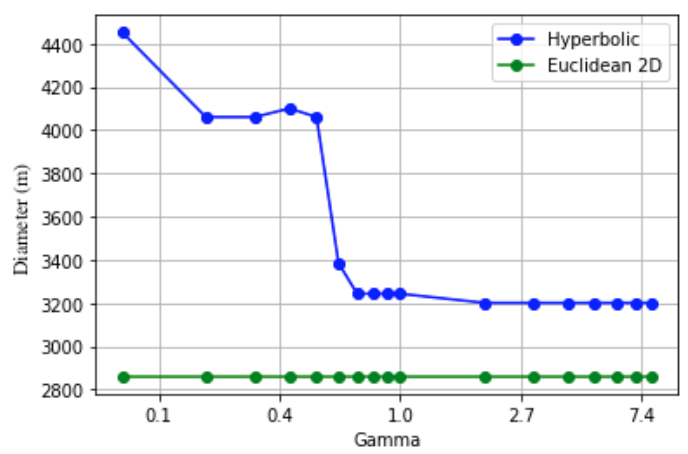

Fig 10: Variation of the clusters diameters according to parameter $\gamma$.

\subsubsection{The number of clusters $K$}

We run all three K-means algorithms (with $H 3, E 2$ and $E 3$ ) for various values of $K(\gamma=1)$, and plot the performance metrics in Fig. 11. We observe that the utilization decreases as $K$ increases, whereas the cost increases with $K$. Both metrics tend to 1 for large $K$. This is explained by the fact that the higher the number of clusters, the more the clustering performance resembles that of the traditional RAN architecture. On the other hand, smaller numbers of clusters $K$ have better performance, but their diameter explodes (non-feasible in practice). For values around $K=6$ the benefits are still considerable. 


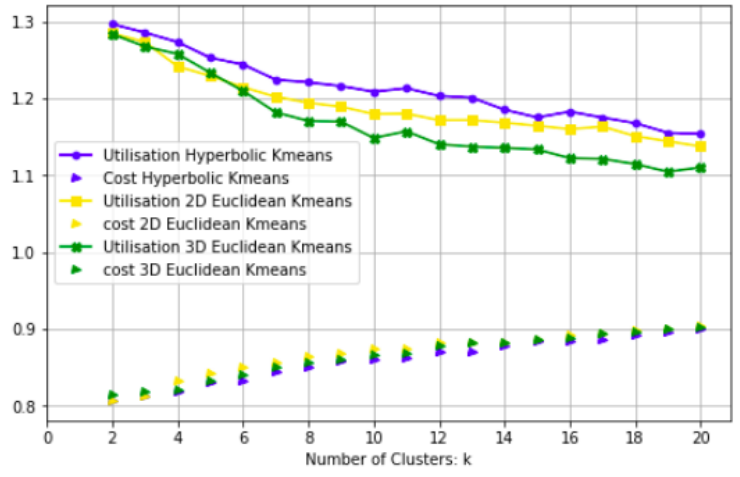

(a) Lille

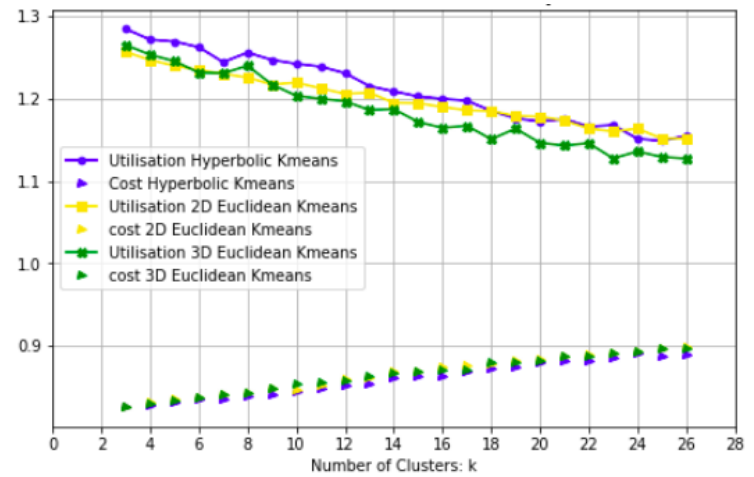

(b) Nantes

Fig 11: Utilization and Cost of the K-means clustering using 3D-Hyperbolic, 2D-Euclidean and 3D-Euclidean distances with varying $K$.

Fig. 12 plots the maximal cluster diameter per partition for each of the three clustering schemes, obtained for different values of $K$. We notice that for $K>5$, the maximal cluster diameter (Lille) is less than $3 \mathrm{~km}$. We choose, for the rest of the evaluations, $K \in[5,10]$ which is a good compromise between the distance threshold and the traffic Utilization/deployment Cost metrics.

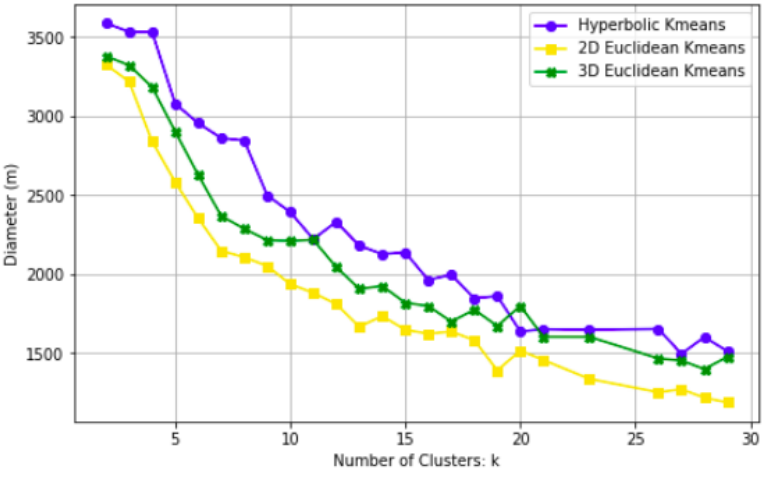

(a) Lille

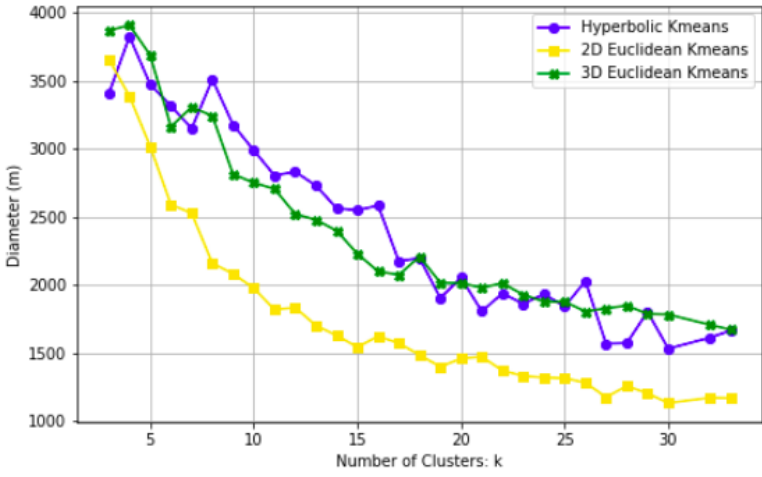

(b) Nantes

Fig 12: Maximal cluster diameter for k-means clustering using Hyperbolic, Euclidean 2D and Euclidean 3D distances according to $\mathrm{K}$.

Take-away 2: The hyperbolic $K$-means outperforms both euclidean schemes in the metrics of utilization and cost throughout the whole range of $K$ values, i.e., for any number of clusters, as Fig. 11a and Fig. 11b show. This improvement in performance is achieved by forming clusters having a diameter comparable to that of 3D-euclidean, and a bit higher than 2D-euclidean, as Fig. 12a and Fig. 12b illustrate. The difference with 2D-euclidean in diameter is of the order of $500 \mathrm{~m}$ for the city of Lille and $1 \mathrm{~km}$ for the city of Nantes. Hence, the hyperbolic K-means spreads a bit more the cluster sizes to achieve higher performance, while keeping them as compact as possible.

\subsection{Comparison over multiple time-slots}

We are now ready to compare the clustering schemes over multiple time-slots. Specifically, for $T=24$ hours, we apply the 3D hyperbolic K-means (H3) with all three features, the 2D- 
Euclidean (E2) K-means that considers only RRHs positions, and finally the DCCA algorithm

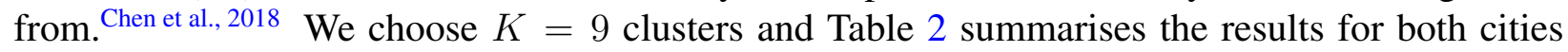
of Lille and Nantes. We observe that the hyperbolic K-means effectively improves the utilization metric by $23 \%$ for Lille and by $25 \%$ for Nantes, while DCCA improves it by $17 \%$ and $19 \%$, respectively. Both methods reduce the cost by around 15\%. The 2D Euclidean K-means achieves an improvement in utilization of $13 \%$ for Lille and $17 \%$ for Nantes, lower than the other two.

Table 2: Comparative Results

\begin{tabular}{|c|c|c|c|c|c|c|}
\hline Method & \multicolumn{2}{|c|}{ Lille City } & \multicolumn{3}{c|}{ Nantes City } \\
\hline & Inputs & Util. & Cost & Inputs & Util. & Cost \\
\hline Hyperbolic $H 3$ & $K=9, \gamma=1, T=24 h$ & 1.23 & 0.85 & $K=9, \gamma=1, T=24 h$ & 1.25 & 0.83 \\
\hline DCCA & Threshold $=3.5 \mathrm{~km}$ & 1.17 & 0.86 & Threshold $=4.7 \mathrm{~km}$ & 1.19 & 0.84 \\
\hline Euclidean $E 2$ & $K=9$ & 1.13 & 0.86 & $K=9$ & 1.17 & 0.88 \\
\hline
\end{tabular}

To compare and better understand the differences between schemes, we illustrate in Fig. 13 the clusters that each method generates for the city of Lille.

Take-away 3: The Hyperbolic K-means over multiple time-slots, produces clusters that are geographically compact, in a similar fashion to the 2D-Euclidean K-means. To better balance the clusters over traffic, the method spreads geographically these clusters while trying to keep them in a compact form, in contrast to the DCCA algorithm.

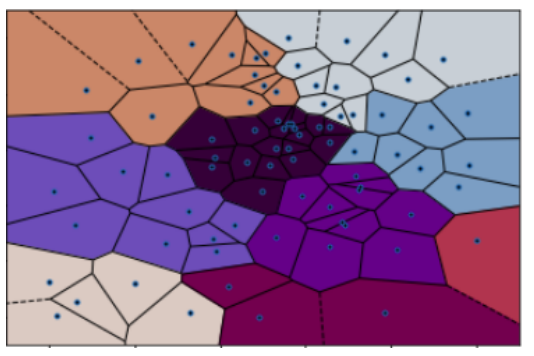

(a) 2D Euclidean K-means.

Utilization: 1.13 , Cost: 0.86

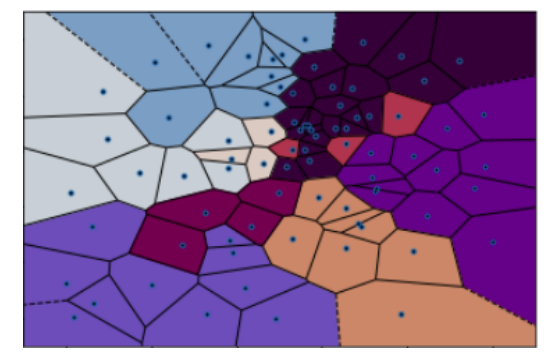

(b) 3D Hyperbolic K-means.

Utilization: 1.23 , Cost: 0.85

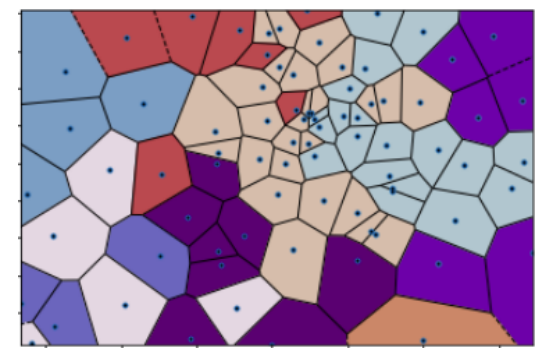

(c) DCCA clustering.

Utilization: 1.17 , Cost: 0.86

Fig 13: Clusters for Lille City with: 2D-Euclidean K-means, 3D-Hyperbolic K-means, and DCCA.

We now vary the number of clusters $K$ and compare the performance between DCCA and the Hyperbolic K-means. First, we run DCCA using various values of the maximum acceptable diameter, because this method does not take the number of clusters $K$ as an input, but instead a threshold on the acceptable diameter to limit the geographical spread of clusters. It generates the number $K$ of formed clusters as output. We use the resulting number $K$ of clusters per realisation as entry to the Hyperbolic K-means, for a fair comparison. The performance results of the two methods for the two cities and in a time-window of 24 hours are illustrated in Fig. 14.

Take-away 4: We observe that in the entire range of $K$, the Hyperbolic K-means offers much higher utilization over time, with a cost similar to the DCCA. This result is stronger than Take-away 2, because now the methods are evaluated over multiple time-slots and DCCA is the state-of-theart. The improvement is more pronounced for a smaller number of clusters (as expected). 
We have discussed throughout the work, that each cluster needs to respect a distant constraint related to the length of the optical fibre. This was used as hard constraint in the input of DCCA. The hyperbolic K-means achieves to produce the same number of clusters with a geographic spread - measured by the diameter Diam - much smaller compared to the DCCA. The comparison of the two methods over Diam is illustrated in Fig. 15. We can read the figure as follows:

Take-away 5: Given some distance constraint (y-axis) the number of clusters $K$ that respect this is considerably smaller in the (robust) Hyperbolic K-means, than in the DCCA. Hence, the $H 3$ method can produce a small number of compact clusters with balanced traffic (high utilization), using the same number of BBU resources (cost) as the DCCA method.

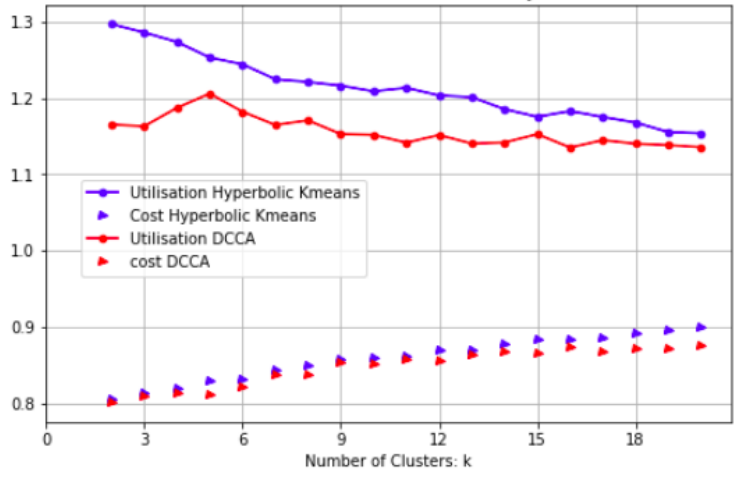

(a) Lille

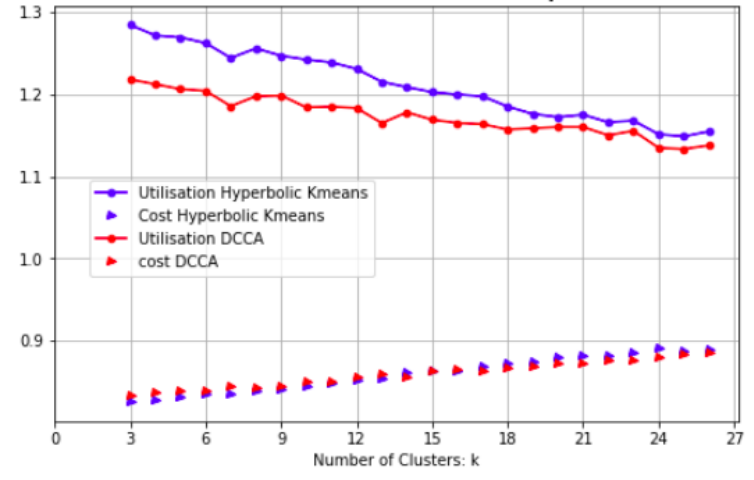

(b) Nantes

Fig 14: Utilization and Cost for Hyperbolic K-means and DCCA over varying number of clusters.

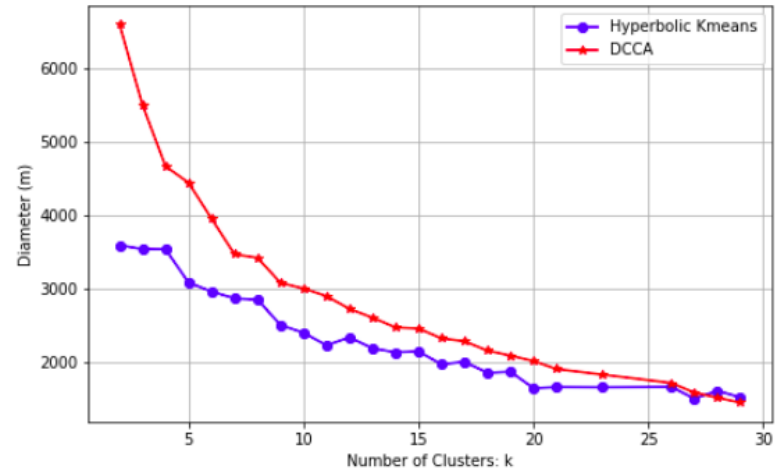

(a) Lille

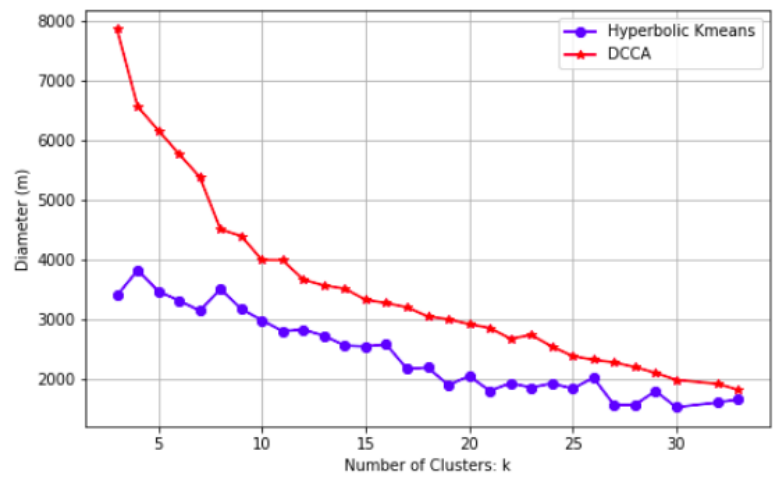

(b) Nantes

Fig 15: Cluster diameters of Hyperbolic K-means and DCCA over varying numbers of clusters.

\section{Conclusion}

In this work, we have addressed one of the challenges in the C-RAN and vRAN architecture, the RU clustering into locally centralised BBU-pools (or RU-CU in the vRAN case). Our objective was to propose a clustering scheme that maximises the resource utilization, that uses reduced number of resources, and respects some distance limitations related to the cluster diameter. To achieve this, we have been based on the vanilla K-means algorithm and proposed a variation that 
considers the traffic (or available resources) as third dimension. Instead of embedding the features in euclidean space, we used the hyperbolic space, because the induced distance has a behaviour that can facilitate the formation of clusters with the desired properties. The resulting clusters from this novel approach further exhibit fairness in the aggregate load. We further made the algorithm robust over multiple time-slots.

To evaluate performance, we have made use of real mobile data from Orange Mobile France, for the cities of Lille and Nantes. Our method effectively reduces deployment cost by $15 \%$ and improves resources utilization by $23-25 \%$. It outperforms both euclidean K-means as well as state-of-the art methods from the literature (DCCA).

Rather noteworthy is the fact that the hyperbolic k-means algorithm proposed in this work has generality. In need not be restricted to problems of RU-BBU association, but could be applied to any clustering problem that deals with various features which need not necessarily be treated homogeneously. It is very useful for all clustering problems that require to group together nodes with complementarity over some feature. The design parameters $\gamma$ and the temporal window of clustering are flexible tuning parameters that allow for performance improvement. The hyperbolic embedding has been proven very useful in this setting, adding further to the arguments in favor of this novel viewpoint.

\section{Acknowledgement}

This work was partially supported by the ANR CANCAN project (ANR-18-CE25-0011).

\section{References}

[Álvarez-Corrales et al., 2017] Álvarez-Corrales, L., Giovanidis, A., Martins, P., and Decreusefond, L. (2017). Wireless node cooperation with resource availability constraints. In 2017 15th International Symposium on Modeling and Optimization in Mobile, Ad Hoc, and Wireless Networks (WiOpt), pages 1-8.

[Boulos et al., 2015] Boulos, K., El Helou, M., and Lahoud, S. (2015). RRH clustering in cloud radio access networks. In 2015 International Conference on Applied Research in Computer Science and Engineering (ICAR), pages 1-6, Lebanon. IEEE.

[Ceselli et al., 2018a] Ceselli, A., Fiore, M., Furno, A., Premoli, M., Secci, S., and Stanica, R. (2018a). Prescriptive analytics for mec orchestration. In 2018 IFIP Networking Conference (IFIP Networking) and Workshops, pages 1-9.

[Ceselli et al., 2018b] Ceselli, A., Fiore, M., Furno, A., Premoli, M., Secci, S., and Stanica, R. (2018b). Prescriptive analytics for mec orchestration. In 2018 IFIP Networking Conference (IFIP Networking) and Workshops, pages 1-9.

[Checko et al., 2015] Checko, A., Christiansen, H. L., Yan, Y., Scolari, L., Kardaras, G., Berger, M. S., and Dittmann, L. (2015). Cloud ran for mobile networks-a technology overview. IEEE Communications Surveys Tutorials, 17(1):405-426. 
[Chen et al., 2017] Chen, L., Liu, L., Fan, X., Li, J., Wang, C., Pan, G., Jakubowicz, J., and Nguyen, T.-M.-T. (2017). Complementary base station clustering for cost-effective and energy-efficient cloud-RAN. In 2017 IEEE SmartWorld, Ubiquitous Intelligence \& Computing, Advanced \& Trusted Computed, Scalable Computing \& Communications, Cloud \& Big Data Computing, Internet of People and Smart City Innovation (SmartWorld/SCALCOM/UIC/ATC/CBDCom/IOP/SCI), pages 1-7, San Francisco, CA. IEEE.

[Chen et al., 2018] Chen, L., Yang, D., Zhang, D., Wang, C., Li, J., and Nguyen, T.-M.-T. (2018). Deep mobile traffic forecast and complementary base station clustering for C-RAN optimization. Journal of Network and Computer Applications, 121:59-69.

[China-Mobile, 2011] China-Mobile (2011). White paper: C-ran the road towards green ran. Technical Report Version 2.5, China Mobile Research Institute.

[China-Unicom, 2017] China-Unicom (2017). China unicom edge computing technology white paper. Technical Report 2017-06, China Unicom Network Technology Research Institute.

[Cisco, 2017] Cisco (2017). White paper: Cisco visual networking index: Global mobile data traffic forecast update, 2017-2022. Technical report, Cisco, San Jose, CA, USA.

[da Silva Coelho et al., 2020] da Silva Coelho, W., Benhamiche, A., Perrot, N., and Secci, S. (2020). On the impact of novel function mappings, sharing policies, and split settings in network slice design. In 2020 16th International Conference on Network and Service Management (CNSM), pages 1-9.

[Decreusefond et al., 2012] Decreusefond, L., Ferraz, E., Martins, P., and Vu, T. T. (2012). Robust methods for lte and wimax dimensioning. In 6th International ICST Conference on Performance Evaluation Methodologies and Tools, pages 74-82.

[Garcia-Saavedra et al., 2018] Garcia-Saavedra, A., Iosifidis, G., Costa-Perez, X., and Leith, D. J. (2018). Joint optimization of edge computing architectures and radio access networks. IEEE Journal on Selected Areas in Communications, 36(11):2433-2443.

[Gerald et al., 2020] Gerald, T., Zaatiti, H., Hajri, H., Baskiotis, N., and Schwander, O. (2020). From node embedding to community embedding : A hyperbolic approach. arXiv:1907.01662.

[Hajri et al., 2019] Hajri, H., Zaatiti, H., Hébrail, G., and Aknin, P. (2019). Apprentissage automatique sur des donnes de type graphe utilisant le plongement de poincaré et les algorithmes stochastiques riemanniens. In Conférence Nationale d'Intelligence Artificielle Année 2019, hal02473573.

[I et al., 2014] I, C.-L., Huang, J., Duan, R., Cui, C., Jiang, J., and Li, L. (2014). Recent Progress on C-RAN Centralization and Cloudification. IEEE Access, 2:1030-1039.

[James et al., 2013] James, G., Witten, D., Hastie, T., and Tibshirani, R. (2013). An Introduction to Statistical Learning, volume 103 of Springer Texts in Statistics. Springer New York, New York, NY.

[Krioukov et al., 2010] Krioukov, D., Papadopoulos, F., Kitsak, M., Vahdat, A., and Boguñá, M. (2010). Hyperbolic geometry of complex networks. Phys. Rev. E, 82:036106.

[Lin et al., 2010] Lin, Y., Shao, L., Zhu, Z., Wang, Q., and Sabhikhi, R. K. (2010). Wireless network cloud: Architecture and system requirements. IBM Journal of Research and Development, 54(1):4:1-4:12.

[Loustau, 2020] Loustau, B. (2020). Hyperbolic geometry. arXiv:2003.11180. 
[Maeder et al., 2014] Maeder, A., Lalam, M., De Domenico, A., Pateromichelakis, E., Wbben, D., Bartelt, J., Fritzsche, R., and Rost, P. (2014). Towards a flexible functional split for cloud-ran networks. In 2014 European Conference on Networks and Communications (EuCNC), pages $1-5$.

[Nickel and Kiela, 2017] Nickel, M. and Kiela, D. (2017). Poincaré embeddings for learning hierarchical representations. In Proceedings of the 31st International Conference on Neural Information Processing Systems, NIPS'17, page 63416350, Red Hook, NY, USA. Curran Associates Inc.

[Wang et al., 2016] Wang, X., Thota, S., Tornatore, M., Chung, H. S., Lee, H. H., Park, S., and Mukherjee, B. (2016). Energy-efficient virtual base station formation in optical-access-enabled cloud-ran. IEEE Journal on Selected Areas in Communications, 34(5):1130-1139.

[Wang et al., 2017] Wang, X., Wang, L., Elayoubi, S. E., Conte, A., Mukherjee, B., and Cavdar, C. (2017). Centralize or distribute? a techno-economic study to design a low-cost cloud radio access network. In 2017 IEEE International Conference on Communications (ICC), pages 1-7.

[Wu et al., 2015] Wu, J., Zhang, Z., Hong, Y., and Wen, Y. (2015). Cloud radio access network (C-RAN): a primer. IEEE Network, 29(1):35-41.

[Yu et al., 2020] Yu, H., Musumeci, F., Zhang, J., Xiao, Y., Tornatore, M., and Ji, Y. (2020). Du/cu placement for c-ran over optical metro-aggregation networks. In Tzanakaki, A., Varvarigos, M., Muñoz, R., Nejabati, R., Yoshikane, N., Anastasopoulos, M., and Marquez-Barja, J., editors, Optical Network Design and Modeling, pages 82-93, Cham. Springer International Publishing. 